\title{
Increased Use of Therapeutic Hypothermia in Infants with Milder Neonatal Encephalopathy due to Presumed Perinatal Asphyxia
}

\author{
Corline E.J. Parmentier ${ }^{a}$ Linda S. de Vries ${ }^{a, b}$ Mona C. Toet ${ }^{a, b}$ \\ Ingrid C. van Haastert ${ }^{a, b}$ Corine Koopman ${ }^{a, b}$ Lauren C. Weeke ${ }^{a, b}$ \\ Floris Groenendaal ${ }^{a, b}$ \\ ${ }^{a}$ Department of Neonatology, Wilhelmina Children's Hospital, University Medical Center Utrecht and \\ Utrecht University, Utrecht, The Netherlands; ${ }^{b}$ UMC Utrecht Brain Center, Utrecht, The Netherlands
}

\section{Keywords}

Neonatal encephalopathy · Therapeutic hypothermia .

Neurodevelopment · Perinatal asphyxia · Neuromonitoring

\begin{abstract}
Introduction: Adverse outcomes have been reported in infants with mild neonatal encephalopathy (NE). Increasing clinical experience with the application of therapeutic hypothermia (TH) may have resulted in the treatment of newborns with milder NE during recent years. Objective: To determine whether infants treated with $\mathrm{TH}$ in the initial years following implementation had a higher degree of NE than infants treated during subsequent years. Methods: Infants with NE treated with TH from February 2008 until July 2017 were included. Thompson and Sarnat scores, amplitude-integrated electroencephalography (aEEG) background patterns before the start of $\mathrm{TH}$, and neurodevelopmental outcome at 2 years were compared between infants treated from February 2008 until October 2012 (period 1) and infants treated from November 2012 until July 2017 (period 2). $R \boldsymbol{e}$ sults: 211 newborns with NE were treated with TH (period 1: $n=109$, period 2: $n=102$ ). Sarnat scores in period 1 and 2 were mild in 7.3 vs. $28.4 \%$, moderate in 66.1 vs. $44.1 \%$, and severe in 26.6 vs. $22.5 \%$, respectively ( $p=0.008$ ). Thompson scores were lower in period 2 (median $=9$, IQR 7-12) than in
\end{abstract}

karger@karger.com www.karger.com/neo

Karger $\frac{1}{\%}$

GOPEN ACCESS
(C) 2020 The Author(s)

Published by S. Karger AG, Basel

This article is licensed under the Creative Commons AttributionNonCommercial-NoDerivatives 4.0 International License (CC BYNC-ND) (http://www.karger.com/Services/OpenAccessLicense). Usage and distribution for commercial purposes as well as any distribution of modified material requires written permission. period $1($ median $=10$, IQR 8.5-13.5, $p=0.018)$. The aEEGs and neurodevelopmental outcomes were comparable between the periods. Conclusions: Based on Thompson and Sarnat scores, but not aEEG background patterns, infants treated during the second period had milder NE than infants treated during the first years following implementation of $\mathrm{TH}$. There was no difference in 2 years neurodevelopmental outcome. Further research is necessary to evaluate the value of TH for infants with clinically mild NE.

(c) 2020 The Author(s) Published by S. Karger AG, Basel

\section{Introduction}

Neonatal encephalopathy (NE) due to presumed perinatal asphyxia is one of the leading causes of neonatal death and morbidity [1]. Since the first randomized controlled trial (RCT) on therapeutic hypothermia (TH) in infants with NE was published in 2005 [2], several studies have shown that $\mathrm{TH}$ started $<6 \mathrm{~h}$ after birth reduces death and disability in term infants with moderate to severe NE. In 2013, a systematic review including 11 RCTs highlighted the neuroprotective effect of this intervention [3].

In the Netherlands $\mathrm{TH}$ has been used in all neonatal intensive care units (NICUs) since 2008 [4]. In 2013, a paper on the implementation of TH in the Netherlands and 
Flanders reported a successful introduction of this intervention with results similar to the major trials [5]. Currently, $\mathrm{TH}$ is the only clinically available therapy proven to be effective in infants with moderate to severe NE [6].

Although historically infants with mild NE were considered to be at minimal risk for adverse neurodevelopmental outcomes [7], recent studies have reported that these infants are at increased risk of adverse outcomes such as behavioral problems and neurodevelopmental impairment $[8,9]$. The role of TH for these babies is unclear. There are no RCTs on TH in this population, and data from studies including these neonates showed variable results. A recent systematic review showed that there is insufficient evidence for a beneficial role of TH in babies with mild NE [10].

Increasing clinical experience and positive results from studies on $\mathrm{TH}$, in combination with studies reporting adverse outcomes in infants with mild NE, may have led towards the cooling of newborns with milder NE over time. The aim of this study was to determine whether infants treated with TH during the initial years after the implementation of this neuroprotective intervention in our hospital had a higher degree of NE than infants cooled during subsequent years.

\section{Materials and Methods}

\section{Subjects}

In this retrospective study, data from infants with clinical signs of NE due to presumed perinatal asphyxia born between February 2008 and August 2017 and treated with hypothermia at the level III NICU of the Wilhelmina Children's Hospital, Utrecht, The Netherlands, were analyzed. Clinical signs of perinatal asphyxia were defined as an Apgar score at $5 \mathrm{~min} \leq 5$, resuscitation, mechanical ventilation during $\geq 10$ min postpartum, $\mathrm{pH}<7.0, \mathrm{BE}$ $<-16 \mathrm{mmol} / \mathrm{L}$, or lactate $>10.0 \mathrm{mmol} / \mathrm{L}$ in umbilical cord blood gas analysis or arterial, venous, or capillary blood gas analysis within $1 \mathrm{~h}$ after delivery.

In our NICU, term infants with well-documented perinatal asphyxia are eligible for $\mathrm{TH}<6 \mathrm{~h}$ after birth when there is encephalopathy based on a Thompson score $>7$, a discontinuous normal voltage pattern or worse on amplitude-integrated electroencephalography (aEEG), or presence of convulsions. For the purpose of this study, infants who were cooled but did not fulfill these criteria were also included. Infants with congenital malformations or syndromes were excluded.

\section{Data Collection}

Medical files were reviewed for demographics. Some data of these infants have been published in previous studies [11-16]. The severity of NE was assessed by analysis of the aEEG background patterns in the hour before start of hypothermia and clinical grading tools documented in the electronic health care system, and a comparison was made between infants treated from February 2008 until October 2012 (period 1) and those treated from November 2012 until July 2017 (period 2).

A two-channel aEEG (F4-P4, F3-P3) was recorded with the BRM2 and BRM3 BrainZ (Natus, Seattle, USA), NicoletOne (Natus, Seattle, USA) and Nihon Kohden (Tokyo, Japan) monitors. The aEEG background pattern in the hour before initiation of hypothermia was scored in 1 of the 5 categories of the HellströmWestas classification system [17].

The Thompson encephalopathy score [18] on admission and the Sarnat score [19] determined after $24 \mathrm{~h}$ of hypothermia were used as clinical tools to assess the severity of NE in the infants.

Adverse outcomes were considered as death, severe hearing or visual impairment, cerebral palsy (CP), or an adverse neurodevelopmental score (Bayley Scales of Infant and Toddler Development-Third Edition (Bayley-III-NL) motor composite score or cognitive composite score $<85$ ) at 2 years of age. For infants in whom the Bayley-III-NL motor composite and cognitive score could not be determined at 2 years of age, a developmental quotient $<88$ tested with the Griffiths Mental Development Scales (GMDS) at 18 months of age was considered as an adverse neurodevelopmental score.

\section{Statistical Analysis}

The data were analyzed using IBM SPSS statistics 25 (Chicago, IL, USA). Differences in clinical variables and in the severity of NE were compared between the periods using the Student $t$ test for normally distributed continuous variables, and Mann-Whitney $U$ tests for continuous variables with non-normal distributions. Nominal dichotomous variables were compared using the $\chi^{2}$ tests. To compare the proportion of infants with mild NE according to Sarnat between the periods, Sarnat scores were dichotomized into "mild" and "moderate-severe" and analyzed using $\chi^{2}$ tests. With a sample of more than 100 patients per time period, we would be able to demonstrate an increase in the proportion of patients with a mild encephalopathy from 10 to $25 \%$ with an alpha of 0.05 and a power of 0.80 .

\section{Results}

\section{Clinical Data}

From February 2008 through July 2017, 211 infants were treated with TH. Table 1 shows the patient characteristics for both periods.

aEEG

Figure 1 shows the distribution of the aEEG background patterns per period. In period 2, a greater proportion of infants (48\%) had a discontinuous normal voltage or continuous normal voltage pattern than in period 1 (36\%), but this difference was not significant $(p=0.08)$. Ten aEEGs were not scored: 7 were not available for analysis, and 3 could not be scored because of artefacts $(n=$ $1)$, status epilepticus $(n=1)$, or gasping $(n=1)$ in the hour before initiation of hypothermia. 
Table 1. Patient characteristics per period

\begin{tabular}{|c|c|c|c|}
\hline & $\begin{array}{l}\text { Period } 1 \\
(n=109)\end{array}$ & $\begin{array}{l}\text { Period } 2 \\
(n=102)\end{array}$ & $p$ value \\
\hline Gestational age, weeks ${ }^{\mathrm{a}}$ & $40.0(39.1-41.0)$ & $39.9(38.4-41.0)$ & 0.304 \\
\hline Birth weight, $g$ & $3,450(3,062-3,835)$ & $3,500(3,039-3,889)$ & 0.428 \\
\hline $\mathrm{pH}^{\mathrm{b}}$ & $6.87(6.77-6.99)$ & $6.90(6.75-7.04)$ & 0.616 \\
\hline Sex, $n(\%)$ & & & 0.918 \\
\hline Male & $58(53.2)$ & $55(53.9)$ & \\
\hline Female & $51(46.8)$ & $47(46.1)$ & \\
\hline Apgar score at $5 \min ^{c}$ & $3(2-5)$ & $3(1-5)$ & 0.353 \\
\hline Death prior to discharge, $n(\%)$ & $37(33.9)$ & $28(27.5)$ & 0.307 \\
\hline Sarnat score, $n(\%)$ & & & 0.008 \\
\hline Mild & $8(7.3)$ & $29(28.4)$ & \\
\hline Moderate & $72(66.1)$ & $45(44.1)$ & \\
\hline Severe & $29(26.6)$ & $23(22.5)$ & \\
\hline Not documented & $0(0)$ & $5(4.9)$ & \\
\hline aEEG, $n(\%)$ & & & 0.230 \\
\hline CNV & $19(17.4)$ & $13(12.7)$ & \\
\hline DNV & $20(18.3)$ & $36(35.3)$ & \\
\hline BS & $35(32.1)$ & $31(30.4)$ & \\
\hline CLV & $8(7.3)$ & $1(1.0)$ & \\
\hline FT & $21(19.3)$ & $17(16.7)$ & \\
\hline No score & $6(5.5)$ & $4(3.9)$ & \\
\hline Thompson score on admission, $n(\%)$ & & & 0.001 \\
\hline$\leq 7$ & $14(12.8)$ & $30(29.4)$ & \\
\hline$>7$ & $91(83.5)$ & $59(57.9)$ & \\
\hline Not documented & $4(3.7)$ & $13(12.7)$ & \\
\hline Bayley-III-NL cognitive composite score ${ }^{\mathrm{d}}$ & $105(100-115)$ & $105(96-120)$ & 0.512 \\
\hline Bayley-III-NL motor composite score $\mathrm{e}^{\mathrm{e}}$ & $112(104-121)$ & $110.5(101-118)$ & 0.161 \\
\hline Adverse outcome $e^{c}, n(\%)$ & $49(45.0)$ & $37(32.0)$ & 0.221 \\
\hline
\end{tabular}

Values are given as medians (with IQR in parentheses) or as $n$ (\%). ${ }^{\text {a }}$ Data available for 208 infants. ${ }^{b}$ Data available for 173 infants. ${ }^{c}$ Data available for 206 infants. ${ }^{d}$ Data available for 130 infants. ${ }^{e}$ Data available for 125 infants. IQR, interquartile range; aEEG, amplitude-integrated electroencephalography; CNV, continuous normal voltage; DNV, discontinuous normal voltage; BS, burst suppression; CLV, continuous low voltage; FT, flat trace.

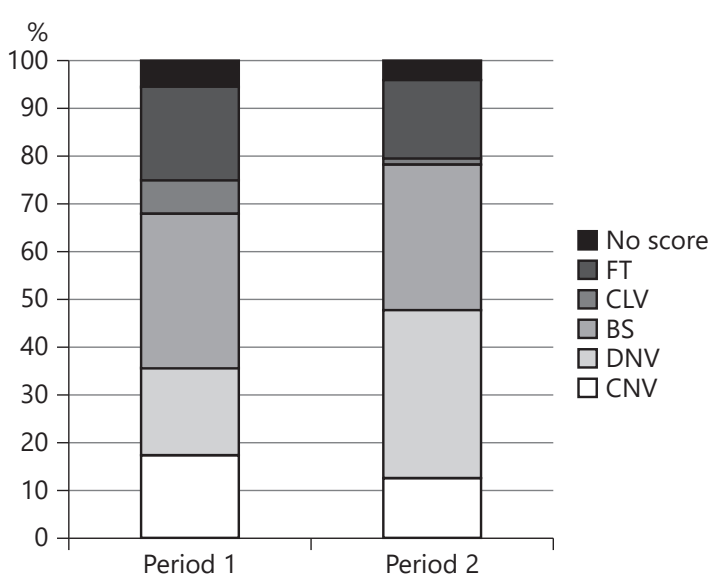

Fig. 1. aEEG background patterns per period. CNV, continuous normal voltage; DNV, discontinuous normal voltage; BS, burstsuppression; CLV, continuous low voltage; FT, flat trace. The difference between period 1 and 2 was not significant $(p=0.08)$.

\section{Thompson Score}

Box plots of the Thompson scores per period are shown in Figure 2. The scores were significantly lower in the second period (median $=9, \mathrm{IQR} 7-12$ ) than in period 1 (median $=10$, IQR $8.5-13.5, p=0.018)$. For 17 infants, Thompson scores were not documented on admission, and for 2 , the scores were not available due to muscle paralysis.

\section{Sarnat Score}

Figure 3 shows the distribution of the Sarnat scores per period. The proportion of newborns with mild NE was significantly higher in the second period $(28.4 \%)$ compared to period $1(7.3 \%, p<0.001)$.

\section{Hypothermia in Spite of a Thompson Score below 8}

Forty-two infants had a Thompson score $\leq 7$ on admission. Table 2 summarizes the reasons for starting $\mathrm{TH}$. Eight of these neonates had an adverse outcome: 4 infants 
Table 2. Reason for start of hypothermia despite Thompson $\leq 7$ per period

\begin{tabular}{llc}
\hline & $\begin{array}{l}\text { Period 1 } \\
(n=14)\end{array}$ & $\begin{array}{l}\text { Period 2 } \\
(n=28)\end{array}$ \\
\hline Clinical seizures or abnormal neurological assessment & $3(21)$ & $9(32)$ \\
DNV or worse on aEEG & $5(36)$ & $11(39)$ \\
Severe abnormalities in the umbilical cord blood analysis* & $1(7)$ & $1(4)$ \\
High lactate level in blood analysis** & $0(0)$ & $1(4)$ \\
Not specified & $5(36)$ & $6(21)$ \\
\hline
\end{tabular}

Values are given as $n(\%) .{ }^{*} \mathrm{pH}<7$ or lactate $>10.0 \mathrm{mmol} / \mathrm{L}$; ${ }^{* *}$ lactate $>10 \mathrm{mmol} / \mathrm{L}$. DNV, discontinuous normal voltage; aEEG, amplitude-integrated electroencephalography.

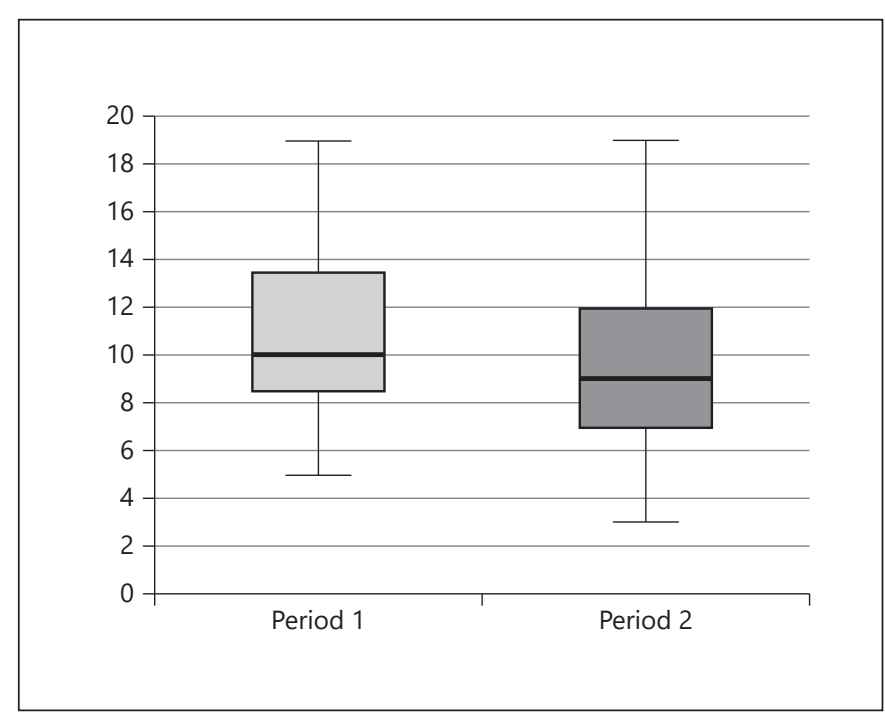

Fig. 2. Box plots of Thompson scores per period. The difference between period 1 and 2 was significant $(p=0.018)$.

died before discharge, 1 developed CP, 2 had a Bayley-IIINL cognitive or motor composite score $<85$ at 2 years age, and 1 had a GMDS $<88$ at 18 months.

\section{Outcome}

The outcome was documented for 206 newborns. There was no significant difference in death rate between the periods. Bayley-III-NL cognitive composite scores were documented for 130 infants (period 1: $n=66$, period 2: $n=64$ ). Bayley-III-NL motor composite scores were documented for 125 infants (period 1: $n=67$, period 2: $n=58$ ). The scores of the infants treated in the initial period were comparable to the scores of the infants treated in period 2 .

Trends in Use of Therapeutic Hypothermia

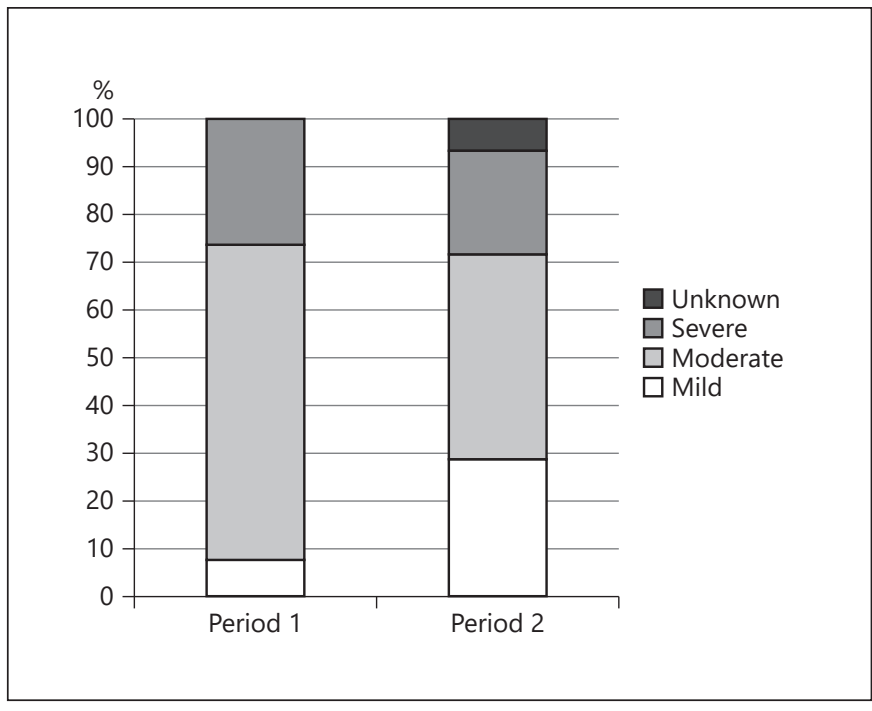

Fig. 3. Sarnat scores per period. The difference between period 1 and 2 was significant $(p=0.008)$.

An adverse outcome was less common among infants cooled in period $2(32 \%)$ than among those cooled in period $1(45 \%)$, but this was not significant $(p=0.22)$. Five infants developed CP (period 1: $n=4$, period 2: $n=1$ ). Five infants had severe hearing or visual impairment (period 1: $n=3$, period 2: $n=2$ ). In seven infants who did not have Bayley-III-NL scores, a GMDS was documented at 18 months of age. Two infants, both cooled in period 2 , had a score $<88$.

For 36 of the infants with mild NE according to Sarnat, the outcome was documented. Three babies had an adverse outcome: 2 had a Bayley-III-NL cognitive composite score of $<85$ at 2 years age and 1 had a GMDS $<88$ at 18 months. 


\section{Discussion}

This study demonstrates that Thompson scores were significantly higher and mild NE, according to the Sarnat scores, was significantly less common during the initial years following initiation of hypothermia compared to following years. The aEEGs of newborns treated in the first period were more depressed compared to those in the second period, but these differences did not reach statistical significance. An adverse outcome was less common in the second period, but this difference was not statistically significant.

Based on the clinical grading tools, the results confirm our hypothesis that infants treated with $\mathrm{TH}$ during the second period had milder NE than infants cooled in the initial years. Several factors may have contributed to this shift. Increasing clinical experience in the use of $\mathrm{TH}$ and positive results reported in studies concerning this intervention may have lowered the threshold for the application of TH. In 2013, a systematic review confirming the neuroprotective role of TH in term and late preterm newborns with moderate to severe NE was published, and in the same year, a paper reported on the successful introduction and absence of significant side effects of $\mathrm{TH}$ in the Netherlands and Flanders $[3,5]$. For neonatologists, the absence of significant side effects is likely to be an important contributor for the decision to start $\mathrm{TH}$ in infants who did not meet all criteria. Moreover, there is increasing evidence against the historical assumption that newborns with mild NE are at low risk for adverse outcomes. A recent review showed that about $25 \%$ of all neonates with mild NE have an abnormal outcome defined as death or motor or cognitive delay at follow-up to 18 months [10]. Finder et al. [9] reported that infants with mild NE had lower cognitive composite scores at 2 years of age compared to controls, and that there was no difference in the mean cognitive composite scores between untreated newborns with mild NE and surviving infants with moderate NE treated with TH. Murray et al. [20] reported that survivors of mild NE had higher rates of disability at 5 years than their peers with uneventful perinatal periods and found similar cognitive outcomes in these children to those with uncooled moderate NE. Another study demonstrated that neonates with mild NE were at increased risk for behavioral problems at school age [21]. As currently $\mathrm{TH}$ is the only clinically available therapy for infants with NE, this may be a reason for clinicians to offer $\mathrm{TH}$ to newborns with milder NE.

Other studies have shown a similar trend in the use of TH in infants with mild NE. Kracer et al. [22] reported an increasing trend in the treatment of newborns with mild NE in a study in California. While in $201038 \%$ of all newborns with mild NE were cooled, this increased to $53 \%$ in 2011 and 55\% in 2012. A national cohort study in Canada showed that $36 \%$ of the neonates admitted to tertiary NICUs with mild NE received TH [23]. There was an increase in the proportion of neonates with mild NE who received $\mathrm{TH}$ over the years. Treatment of mild NE with $\mathrm{TH}$ was associated with lower odds of brain injury on MRI, but selection bias could not be ruled out. A study by Mehta et al. [24] on 207 infants who received TH reported that $23 \%$ of these infants were documented to only have features of mild NE. A national survey in the UK showed that $75 \%$ of the included centers offered $\mathrm{TH}$ for infants with mild encephalopathy [25]. Gagne-Loranger et al. [26] reported that $10 \%$ of the 128 infants that were cooled in their NICU between 2008 and 2012 had mild NE. These infants were cooled because of aEEG findings consistent with moderate encephalopathy, suggesting that aEEG was preferred over clinical criteria in the selection of infants for TH. In a meta-analysis including 13 observational studies, $22 \%$ of the cooled infants had mild NE [1].

Despite the limited side effects of TH reported in trials [3], it is important to realize that $\mathrm{TH}$ results in an increased use of invasive treatments and the separation of infants from their parents, with a delay in oral (breast) feeding and impairment of parent-infant bonding. Current studies on the value of TH in neonates with mild NE are inconclusive. In a study by Montaldo et al. [27], neonates with mild NE had improved MR spectroscopy biomarkers and reduced white matter injury on MRI after $\mathrm{TH}$, suggesting a neuroprotective role of cooling in these babies. However, the mean cognitive, motor, and language scores at 2 years were comparable between cooled and non-cooled neonates. In another study, $40 \%$ of the non-cooled infants who had mild NE and available brain MRI results developed brain injury, while among the cooled infants with mild NE $31 \%$ developed brain injury [26]. A recent systematic review concluded that there is insufficient evidence for a beneficial role of $\mathrm{TH}$ in mild NE [10].

The fact that in our study the aEEG background patterns were not significantly different between the periods may indicate that in the second period aEEG played a more important role in the decision to start $\mathrm{TH}$ compared to the Thompson score, which may be confirmed by the higher percentage of infants cooled because of an abnormal aEEG background pattern despite a Thompson score $\leq 7$ in the second period than in period 1 . In our NICU, 
in infants presenting with a Thompson score just below 8 and abnormalities on aEEG, a decision to cool was based on the aEEG. Despite a comparable reliability in assessing the severity of NE shown in a previous paper [11], aEEG may be preferable because of the continuous measurement, its ability to identify subclinical seizures, and the possibility for a rapid expert revision. Furthermore, aEEG was used to select infants for TH in two multicenter trials on $\mathrm{TH}[2,28]$.

Despite the fact that an adverse outcome was less common among the children who were cooled in the second period, no significant difference between the two groups could be demonstrated. Whether there is a difference in outcome at school age still needs to be seen.

Although more infants with mild NE were cooled, the number of newborns cooled in the initial years did not differ from the number of infants cooled in the subsequent period. This may be due to improvements in perinatal care over the years, as is reflected by the substantial difference in the proportion of infants with moderate and severe NE between the two time periods.

Strengths of our study include the large sample size, a study period of roughly 10 years, and details regarding the neurodevelopmental outcome of these infants at 2 years. NE was carefully graded using both aEEG and clinical tools, and a detailed description of the reason for start of $\mathrm{TH}$ was given for newborns who were not eligible for this intervention based on their Thompson score.

Limitations of this study include the retrospective nature and the restriction to a single center. For several newborns, the clinical scores were not documented, and some aEEGs were not available for analysis or were unreliable because of seizures or artefacts.

In summary, this study demonstrated a trend towards cooling newborns with milder NE based on clinical grading tools. There was no significant difference in the aEEG and outcomes at 2 years between the early and later years. Our data highlight the need for well-designed studies to evaluate the neuroprotective role of $\mathrm{TH}$ for newborns with clinically mild NE.

\section{Acknowledgement}

The authors thank the neonatologists, nurses, and physician assistants of the Neonatal Intensive Care Unit for their devoted use of the aEEG, and the psychologists and physical therapists for their efforts in the follow-up of the infants.

\section{Statement of Ethics}

The Ethical Review Board of the University Medical Center Utrecht waived the requirement for informed consent for this study with anonymized clinical data.

\section{Conflict of Interest Statement}

The authors have no conflicts of interest to declare.

\section{Funding Sources}

Part of this study was funded by an unrestricted grant from the Stichting Neonatale Neurologie Utrecht (www.snnu.nl).

\section{Author Contributions}

L.S.d.V., F.G., and C.E.J.P.: study conception and design. L.S.d.V., L.C.W., M.C.T., I.C.H., C.K., F.G., and C.E.J.P.: acquisition of data. C.E.J.P, L.S.d.V., and F.G.: analysis and interpretation of data. C.E.J.P., L.S.d.V., L.C.W., and F.G.: drafting of manuscript. All authors: critical revision.

\section{References}

1 Saw CL, Rakshasbhuvankar A, Rao S, Bulsara M, Patole S. Current practice of therapeutic hypothermia for mild hypoxic ischemic encephalopathy. J Child Neurol. 2019 Jun;34(7): 402-9.

2 Gluckman PD, Wyatt JS, Azzopardi D, Ballard R, Edwards AD, Ferriero DM, et al. Selective head cooling with mild systemic hypothermia after neonatal encephalopathy: multicentre randomised trial. Lancet. 2005 Feb; 365(9460):663-70.

3 Jacobs SE, Berg M, Hunt R, Tarnow-Mordi WO, Inder TE, Davis PG. Cooling for newborns with hypoxic ischaemic encephalopa- thy. Cochrane Database Syst Rev. 2013 Jan;(1): CD003311.

4 de Haan TR, Bijleveld YA, van der Lee JH, Groenendaal F, van den Broek MP, Rademaker CM, et al. Pharmacokinetics and pharmacodynamics of medication in asphyxiated newborns during controlled hypothermia. The PharmaCool multicenter study. BMC Pediatr. 2012 May;12(1):45.

5 Groenendaal F, Casaer A, Dijkman KP, Gavilanes AW, de Haan TR, ter Horst HJ, et al. Introduction of hypothermia for neonates with perinatal asphyxia in the Netherlands and Flanders. Neonatology. 2013;104(1):15-21.
6 Burnsed J, Zanelli SA. Neonatal therapeutic hypothermia outside of standard guidelines: a survey of U.S. neonatologists. Acta Paediatr. 2017 Nov; 106(11):1772-9.

7 El-Dib M, Inder TE, Chalak LF, Massaro AN, Thoresen M, Gunn AJ. Should therapeutic hypothermia be offered to babies with mild neonatal encephalopathy in the first $6 \mathrm{~h}$ after birth? Pediatr Res. 2019 Mar;85(4):442-8.

8 Conway JM, Walsh BH, Boylan GB, Murray DM. Mild hypoxic ischaemic encephalopathy and long term neurodevelopmental outcome - a systematic review. Early Hum Dev. 2018 May;120:80-7. 
9 Finder M, Boylan GB, Twomey D, Ahearne C, Murray DM, Hallberg B. Two-year neurodevelopmental outcomes after mild hypoxic ischemic encephalopathy in the era of therapeutic hypothermia. JAMA Pediatr. 2019 Nov;174(1):48-55.

10 Kariholu U, Montaldo P, Markati T, Lally PJ, Pryce R, Teiserskas J, et al. Therapeutic hypothermia for mild neonatal encephalopathy: a systematic review and meta-analysis. Arch Dis Child Fetal Neonatal Ed. 2020 Mar; 105(2):225-8

11 Weeke LC, Vilan A, Toet MC, van Haastert IC, de Vries LS, Groenendaal F. A Comparison of the Thompson encephalopathy score and amplitude-integrated electroencephalography in infants with perinatal asphyxia and therapeutic hypothermia. Neonatology. 2017; 112(1):24-9.

12 Weeke LC, Groenendaal F, Mudigonda K, Blennow M, Lequin MH, Meiners LC, et al. A novel magnetic resonance imaging score predicts neurodevelopmental outcome after perinatal asphyxia and therapeutic hypothermia. J Pediatr. 2018 Jan;192:33-40.e2.

13 Alderliesten T, de Vries LS, Staats L, van Haastert IC, Weeke L, Benders MJ, et al. MRI and spectroscopy in (near) term neonates with perinatal asphyxia and therapeutic hypothermia. Arch Dis Child Fetal Neonatal Ed. 2017 Mar;102(2):F147-52.

14 Imai K, de Vries LS, Alderliesten T, Wagenaar $\mathrm{N}$, van der Aa NE, Lequin $\mathrm{MH}$, et al. MRI changes in the thalamus and basal ganglia of full-term neonates with perinatal asphyxia. Neonatology. 2018;114(3):253-60.
15 Diederen CM, van Bel F, Groenendaal F. Complications during therapeutic hypothermia after perinatal asphyxia: a comparison with trial data. Ther Hypothermia Temp Manag. 2018 May;8(4):211-5.

16 Hakobyan M, Dijkman KP, Laroche S, Naulaers G, Rijken M, Steiner K, et al. Outcome of infants with therapeutic hypothermia after perinatal asphyxia and early-onset sepsis. Neonatology. 2019;115(2):127-33.

17 Hellström-Westas L, Rosén I, de Vries LS, Greisen G. Amplitude-integrated EEG classification and interpretation in preterm and term infants. Neoreviews. 2006;7(2):e76-87.

18 Thompson CM, Puterman AS, Linley LL, Hann FM, van der Elst CW, Molteno CD, et al. The value of a scoring system for hypoxic ischaemic encephalopathy in predicting neurodevelopmental outcome. Acta Paediatr. 1997 Jul;86(7):757-61.

19 Shalak LF, Laptook AR, Velaphi SC, Perlman JM. Amplitude-integrated electroencephalography coupled with an early neurologic examination enhances prediction of term infants at risk for persistent encephalopathy. Pediatrics. 2003 Feb;111(2):351-7.

20 Murray DM, O'Connor CM, Ryan CA, Korotchikova I, Boylan GB. Early EEG grade and outcome at 5 years after mild neonatal hypoxic ischemic encephalopathy. Pediatrics. 2016 Oct; 138(4):e20160659.

21 van Handel M, Swaab H, de Vries LS, Jongmans MJ. Behavioral outcome in children with a history of neonatal encephalopathy following perinatal asphyxia. J Pediatr Psychol. 2010 Apr;35(3):286-95.

22 Kracer B, Hintz SR, Van Meurs KP, Lee HC. Hypothermia therapy for neonatal hypoxic ischemic encephalopathy in the state of California. J Pediatr. 2014 Aug;165(2):267-73.
23 Goswami IR, Whyte H, Wintermark P, Mohammad K, Shivananda S, Louis D, et al.; Canadian Neonatal Network Investigators. Characteristics and short-term outcomes of neonates with mild hypoxic-ischemic encephalopathy treated with hypothermia. J Perinatol. 2020 Feb;40(2):275-83.

24 Mehta S, Joshi A, Bajuk B, Badawi N, McIntyre S, Lui K. Eligibility criteria for therapeutic hypothermia: from trials to clinical practice. J Paediatr Child Health. 2017 Mar; 53(3):295-300.

25 Oliveira V, Singhvi DP, Montaldo P, Lally PJ, Mendoza J, Manerkar S, et al. Therapeutic hypothermia in mild neonatal encephalopathy: a national survey of practice in the UK. Arch Dis Child Fetal Neonatal Ed. 2018 Jul; 103(4):F388-90.

26 Gagne-Loranger M, Sheppard M, Ali N, Saint-Martin C, Wintermark P. Newborns referred for therapeutic hypothermia: association between initial degree of encephalopathy and severity of brain injury (what about the newborns with mild encephalopathy on admission?). Am J Perinatol. 2016 Jan;33(2): 195-202.

27 Montaldo P, Lally PJ, Oliveira V, Swamy R, Mendoza J, Atreja G, et al. Therapeutic hypothermia initiated within 6 hours of birth is associated with reduced brain injury on MR biomarkers in mild hypoxicischaemic encephalopathy: a non-randomised cohort study. Arch Dis Child Fetal Neonatal Ed. 2019 Sep;104(5):F515-20.

28 Azzopardi DV, Strohm B, Edwards AD, Dyet L, Halliday HL, Juszczak E, et al.; TOBY Study Group. Moderate hypothermia to treat perinatal asphyxial encephalopathy. N Engl J Med. 2009 Oct;361(14):1349-58. 